\title{
The modal nature of structures in ontic structural realism
}

\author{
Michael Esfeld \\ University of Lausanne, Department of Philosophy \\ CH-1015 Lausanne, Switzerland \\ Michael-Andreas.Esfeld@unil.ch \\ (forthcoming in International Studies in the Philosophy of Science 23 (2009))
}

\begin{abstract}
Ontic structural realism is the view that structures are what is real in the first place in the domain of fundamental physics. The structures are usually conceived as including a primitive modality. However, it has not been spelled out as yet what exactly that modality amounts to. This paper proposes to fill this lacuna by arguing that the fundamental physical structures possess a causal essence, being powers. Applying the debate about causal vs. categorical properties in analytic metaphysics to ontic structural realism, I show that the standard argument against categorical and for causal properties holds for structures as well. Structural realism, as a position in the metaphysics of science that is a form of scientific realism, is committed to causal structures. The metaphysics of causal structures is supported by physics, and it can provide for a complete and coherent view of the world that includes all domains of empirical science.
\end{abstract}

\section{Introduction}

Ontic structural realism is a current in contemporary metaphysics of science that maintains that in the domain of fundamental physics, there are structures in the first place rather than objects with an intrinsic identity. Its main motivation is to develop a tenable version of scientific realism in form of an ontology that meets the challenges of modern physics, giving an account of entanglement in quantum physics and of space-time in the theory of general relativity. The claim is that there are structures of entanglement instead of objects with an intrinsic identity in the domain of quantum physics (Ladyman 1998, French \& Ladyman 2003, Esfeld 2004) and metrical structures, which include the gravitational energy, instead of space-time points with an intrinsic identity in the domain of the theory of general relativity (Esfeld \& Lam 2008; cf. also Slowik 2005).

The founders of ontic structural realism, Steven French and James Ladyman, tend to hold that there are structures all the way down; if there are objects at all, these are derived from the structures as being nodes of structures, instead of structures requiring objects that stand in them (Ladyman 1998, French \& Ladyman 2003, French 2006; but see also the more balanced position in Ladyman \& Ross 2007, ch. 2 to 5). They thus invite the objection that the notion of structures without objects is not intelligible (e.g. Busch 2003 and Psillos 2006, 562-566). However, a more moderate version of ontic structural realism has recently been developed in reply to that objection, proposing that physical structures are networks of concrete, qualitative physical relations among objects that are nothing but what stands in these relations, that is, do not possess an intrinsic identity over and above the relations in which they stand (Esfeld 2004, Esfeld \& Lam 2008, Floridi 2008). This position takes notably the entangled states of elementary quantum systems and the metrical relations among space-time points to be 
concrete structures in this sense. On this more moderate version, no paradox arises, since objects are admitted as standing in the relations. Consequently, there is no need to change standard first order logic. The argumentation of this paper can be based on this moderate version of ontic structural realism, thus avoiding the mentioned stock objection.

The structures to which ontic structural realism is committed have been conceived by James Ladyman as including a primitive modality (Ladyman 1998, Ladyman \& Ross 2007, ch. 2 to 5), and Steven French (2006) expresses sympathy with this view. However, it has not been spelled out as yet what exactly that modality consists in. This paper sets out to fill this lacuna, proposing that the fundamental physical structures are causal structures, accounting for the necessity of the physical laws and giving rise to necessary connections in nature. Drawing on the debate about categorical vs. causal properties in analytic metaphysics, I show that the main argument for causal properties - namely that if the properties were categorical instead of causal, they would underdetermine the causal and nomological relations that there are in the world -, applies to structures as well if they are conceived as being categorical, including no primitive modality. Against that background, I maintain that ontic structural realism, as a position in the metaphysics of science that is a form of scientific realism, is committed to causal structures.

The paper proceeds as follows: first, I briefly recall the main features of the debate about categorical vs. causal properties in analytic metaphysics (section 2). I then work out in what sense the standard argument for causal properties applies to structures as well, thus speaking in favour of conceiving the fundamental physical structures as including a primitive modality and spelling that modality out in causal terms; a further advantage of this view is that it provides for a clear distinction between physical and mathematical structures (section 3 ). Section 4 outlines how it is possible in concrete terms to conceive the structures to which the fundamental physical theories are committed in a causal manner. In conclusion, I sketch out how the ontology of causal structures opens up the way for developing a complete and coherent view of the world that includes all domains of empirical science.

\section{Categorical vs. causal properties in analytic metaphysics}

According to the metaphysics of categorical properties, properties are purely qualitative: what they are is independent of the causal and nomological relations in which they stand. Intrinsic are all and only those properties that an object possesses irrespective of whether or not there are other contingent objects; in brief, having or lacking an intrinsic property is independent of accompaniment or loneliness (Langton \& Lewis 1998 and see Hoffmann 2008, part 1, for a detailed discussion of intrinsic properties). The most prominent conception of a metaphysics of categorical and intrinsic properties is the position known as Humean metaphysics in today's philosophy. Consider David Lewis' thesis of Humean supervenience:

It is the doctrine that all there is to the world is a vast mosaic of local matters of particular fact, just one little thing and then another. (...) We have geometry: a system of external relations of spatio-temporal distance between points. Maybe points of space-time itself, maybe point-sized bits of matter or aether or fields, maybe both. And at those points we have local qualities: perfectly natural intrinsic properties which need nothing bigger than a point at which to be instantiated. For short: we have an arrangement of qualities. And that is all. There is no difference without difference in the arrangement of qualities. All else supervenes on that. (Lewis 1986, ix-x) 
Conceiving the fundamental physical properties as natural, intrinsic and categorical properties, Lewis accepts the whole distribution of these properties in space-time as primitive. It is not only contingent that there is the distribution of fundamental physical properties as a whole that there is in fact, but each element in that distribution is also contingent. Consequently, for each single token of a fundamental physical property at a space-time point, it is conceivable and metaphysically possible to hold that token (or its counterpart) fixed and to vary all the other tokens. The physical properties instantiated at any given space-time point or region do not impose any restrictions at all on the physical properties that can be instantiated at other space-time points or regions (Beebee 2006). There thus are no necessary connections in nature.

If the fundamental physical properties are categorical and intrinsic, how can we know them? Consider how Frank Jackson, who has a favourable attitude towards Humean metaphysics, answers that question:

When physicists tell us about the properties they take to be fundamental, they tell us what these properties $d o$. This is no accident. We know about what things are like essentially through the way they impinge on us and our measuring instruments. ... [This] does suggest the possibility that (i) there are two quite different intrinsic properties, $P$ and $P^{*}$, which are exactly alike in the causal relations they enter into, (ii) sometimes one is possessed and sometimes the other, and (iii) we mistakenly think that there is just one property because the difference does not make a difference (...). An obvious extension of this possibility leads to the uncomfortable idea that we may know next to nothing about the intrinsic nature of the world. We know only its causal cum relational nature. (Jackson 1998, 23-24; see also Blackburn 1990)

We gain knowledge about the world via the causal relations in which what there is in the world stands. Hence, we can specify what there is in the world only down to causal-cumstructural equivalence. Consequently, if what there is in the world are in the last resort fundamental categorical and intrinsic properties, we cannot know these properties insofar as they are categorical and intrinsic. Different arrangements of such properties can all make true the same causal-cum-structural descriptions so that we are in principle unable to discover those differences.

This issue, however, is not only about knowledge. It is at its roots a metaphysical issue, which has certain consequences for epistemology. If the fundamental properties are categorical and intrinsic, then there are worlds that are different because they differ in the distribution of the intrinsic properties that are instantiated in them, although there is no difference in causal and nomological relations and thus no discernible difference between them. This position therefore implies quidditism and humility: the properties possess a primitive qualitative character (quiddity), since what they are is independent of the causal and the nomological relations into which they enter, and we cannot know what the properties are, but only the causal and nomological relations in which they stand (humility). A quidditistic difference between worlds is a qualitative difference (by contrast to a haecceistic difference, concerning the question which individuals there are in a given world) that implies that worlds have to be counted as different although they are indiscernible. A gap between metaphysics postulating primitive qualities - and epistemology thus arises.

Ann Whittle (2006, 469-472) and Alyssa Ney (2007, 50-53) argue against humility being a problematic consequence, highlighting that the causal descriptions are descriptions of the properties in question. However, these descriptions cannot disclose what the properties they 
refer to are, since what these properties are is independent of the causal roles they play in a given world: two different types of properties, say mass and charge, can swap their causal roles in two different worlds, these roles depending on what else there is in a given world. Consequently, the description of the mass-role and the description of the charge-role in the actual world do not tell us anything about the essences of the properties that play these roles in the actual world (see Locke 2009, 227-228). Lewis himself (2009) endorses both the commitments to quidditism and to humility.

However, these are rather uncomfortable commitments, notably for a metaphysics based on empirical science - postulating something that is a primitive quality and that gives rise to differences that do not make a difference (as Jackson puts it in the quotation above) only in order to uphold the view that the properties are categorical and intrinsic. One can avoid these commitments by taking the essence of the properties to be tied to the causal and nomological relations in which they stand (Shoemaker 1980). If what the properties are is tied to certain causal relations, properties are dispositions in the sense of powers: they are the disposition (power) to produce certain effects. If this view holds for the fundamental physical properties as well, it is thus committed to ungrounded dispositions (Mumford 2006): the fundamental physical properties are dispositions without a categorical basis.

Hence, although Humean metaphysics seems to be parsimonious at first glance, it is not that parsimonious after all, postulating that properties have an essence beyond the causal relations into which they enter, that essence consisting in a primitive qualitative character (quiddity) that forces us to count worlds that are indiscernible as different possible worlds. Deleting these commitments leads to the causal theory of properties, namely to the view that there is nothing more to the properties than the power to bring about certain effects, and that view then implies that we have to countenance a primitive modality. However, seen from the perspective of avoiding quidditism and humility, the commitment to a primitive modality in the sense that it is a primitive fact that properties are certain powers is not at all mysterious or ontologically inflationary.

The causal theory of properties is mainly advocated by Sydney Shoemaker (1980) and Alexander Bird (2007) (see also Hawthorne 2001 who calls this view "causal structuralism" and Chakravartty 2007, ch. 3 to 5). There also are intermediate positions: C. B. Martin (1997) and John Heil (2003, ch. 11) are usually read as maintaining that properties are both categorical and dispositional. However, thus read, their view raises the question what the relationship between these two ways of being of properties is and how it avoids the objections of quidditism and humility as regards the categorical way of being of properties (but see the manner in which Heil 2009, 178, describes the last position of C. B. Martin, namely as conceiving properties as "powerful qualities"). Brian Ellis (2001, ch. 1 and 3) holds that only some properties are causal, whereas others are categorical. However, if one is committed to the consequences of Humeanism for some properties, why not endorse these consequences for all properties? And if one is committed to tying the nature of some properties to certain causal relations, why not do so for all properties? I shall sketch out in section 4 how this latter view is compatible with fundamental physics.

There is nothing paradoxical about the causal theory of properties. Properties as powers are not mere potentialities, but real, actual properties. They thus are certain qualities whose nature is to produce certain effects. In other words, causal properties are such that insofar as they are certain qualities, they are powers to produce certain specific effects. To put the matter in 
terms of object talk, qua being a certain way, that is, by having certain qualities, objects have the power to produce other objects, or other properties in objects. Take charge for example: insofar as charge is a qualitative property, distinct from e.g. mass, it is the power to build up an electromagnetic field, resulting in the attraction of opposite-charged and the repulsion of like-charged objects. The Humean and the anti-Humean agree about the properties that there are in the world. They disagree about the ontology of properties - whether properties are pure qualities (quiddities) or whether their being certain qualities consists in the power to produce certain specific effects.

In recent literature, doubts are expressed as to whether in case one conceives the fundamental properties as dispositions and thus in a causal manner, one inevitably is committed to recognizing necessary connections in the world, thereby contradicting Humean metaphysics (Handfield 2008, Anjum \& Mumford forthcoming). Nonetheless, there is a clear contrast between the Humean metaphysics of categorical properties and the metaphysics of causal properties. According to Humean metaphysics, property tokens of the same type can stand in very different causal relations in different possible worlds. By contrast, according to the causal theory of properties, the qualitative character of a property type determines the causal role that the property type in question plays and hence determines the causal relations in which the tokens of the property type in question stand. Since, on that view, it is the nature of the properties to produce certain effects, the connection between cause and effect is a necessary one. Consequently, this theory goes not only with an anti-Humean view of causation but also with an anti-Humean view of the physical laws: these are metaphysically necessary because they flow from the nature of the properties. There is thus no quiddity, for what the properties are consists in the production of certain effects, and no humility, since what the properties are can in principle be discovered via the effects they produce (more precisely, via the effects that objects or events produce in virtue of having certain properties).

If the main reason for adopting the causal theory of properties is to avoid the commitments to quidditism and to humility, then the fundamental properties cannot be regarded as needing outside manifestation conditions for exercising the powers that they are. Otherwise, there could be fundamental properties of two different types $P$ and $P^{*}$ present in the world without that difference showing up anywhere in the world because the appropriate manifestation conditions always lack. Accordingly, there could be two worlds $w$ and $w^{*}$ that are indiscernible as regards the causal and nomological relations, the only difference between them being the fact that $P$ is instantiated in $w$ and $P^{*}$ in $w^{*}$, without that difference showing up anywhere in $w$ or $w^{*}$ because the manifestation conditions for the difference in powers between $P$ and $P^{*}$ always lack in both these worlds. It is unproblematic to conceive the fundamental properties as not needing outside manifestation conditions in order to exercise the powers that they are, since these powers are not mere potentialities, but real, actual properties. The appropriate model are not macroscopic dispositions such as the disposition of water to dissolve sugar, but rather the dispositions of radioactive atoms for spontaneous decay or of particles with charge (field sources) to build up electromagnetic fields.

\section{Multiple realization and the argument from underdetermination}

The debate about categorical vs. causal properties in analytic metaphysics usually takes for granted that the properties are intrinsic. Let us now apply that debate to structures as conceived by ontic structural realism. Structures are properties, too, in a broad sense of the 
notion of properties, namely relations instead of intrinsic properties, requiring more than one object in order to be instantiated. My claim is that the master argument for causal properties and against quidditism and humility rehearsed in the preceding section can be transformed so that it - or something similar to it - applies to fundamental structures as well (nonetheless, it is possible to defend a Humean version of structural realism; see Sparber 2008, ch. 5). Consequently, we get an argument for conceiving the fundamental structures as being causal in themselves, that is, as causal structures. I shall set out that argument in this section and briefly explain in the next section how the commitment to causal structures fits in with current physics.

The argumentation in this and the next section can thus be seen as a reply to Stathis Psillos (2006, 567-570) who maintains that ontic structural realism cannot accommodate causation, and to Anjan Chakravartty (2007, ch. 3 to 5) who accepts the causal theory of properties, but then conceives concrete physical structures as the relations that underlying causal properties produce. The claim of this paper, by contrast, is that the fundamental physical structures are causal in themselves so that there is no need to postulate underlying causal properties (see also French 2006, 181-182, for the possibility of this claim).

Jackson says in the quotation above that we gain knowledge of something only insofar as it stands in a causal relation to our cognitive apparatus, however indirect that relation may be. The structures to which the fundamental physical theories refer cannot stand in a direct causal relation to our cognitive apparatus. They are theoretical entities. The quantum relations of entanglement are not observable. What is observed are certain correlations among measurement outcomes (but no superpositions of such correlations, that is, no entanglement). One recognizes the existence of superpositions including entanglement in order to explain the measurement outcomes and the correlations between them.

By the same token, the spatio-temporal, gravitational structures that the theory of general relativity acknowledges and that show that space-time is curved are not observable as such. A local observer cannot determine whether or not space-time is curved. It is only when one takes the various local observations together in order to form a representation of space-time as a whole that one gets to regard space-time as curved. In short, the fundamental physical structures are theoretical entities, and we recognize them because they explain the observed phenomena. The explanation in question is a causal one: those structures are the causal origin of the observed phenomena.

However, if, as on Humean metaphysics, the properties are categorical and intrinsic, all the causal relations can be the same in two possible worlds, but the underlying fundamental categorical and intrinsic properties different. As Jackson puts it in the quotation above, there is "the possibility that (i) there are two quite different intrinsic properties, $P$ and $P^{*}$, which are exactly alike in the causal relations they enter into, (ii) sometimes one is possessed and sometimes the other, and (iii) we mistakenly think that there is just one property because the difference does not make a difference" (Jackson 1998, 23-24).

We thus face a case of multiple realization: the causal relations can be realized by categorical and intrinsic properties of type $P$, and they can also be realized by categorical and intrinsic properties of type $P^{*}$ without there being any difference in the causal relations. Since according to Humean metaphysics any causal relation depends on the whole distribution of the fundamental physical properties, let us put the point in terms of whole possible worlds: there are two possible worlds $w$ and $w^{*}$, they are indiscernible as regards the causal relations 
that obtain in them, but are distinct in that different underlying fundamental physical categorical and intrinsic properties realize these causal relations. This case of multiple realization contrasts with the familiar cases (such as e.g. the realization of psychological by neurobiological properties) in that there is no difference at all in causal relations so that we can in principle not know the realizing properties. Lewis (2009) therefore speaks of Ramseyan humility: we cannot know what realizes the Ramsey sentence of a world. There are worlds that are described by the same type of Ramsey sentence but that are distinct in that different arrangements of fundamental categorical and intrinsic properties realize the Ramsey sentence in question.

By contrast, if the fundamental properties are intrinsic, but causal properties and if they do not need outside manifestation conditions for exercising the powers that they are, then there is no such multiple realization. It is then not possible that "there are two quite different intrinsic properties, $P$ and $P^{*}, \ldots$ [and] the difference does not make a difference". If the properties are causal, then a difference in the fundamental properties makes a difference in the causal relations that obtain in the world in question. It is then excluded that there are two possible worlds which are indiscernible as regards the effects that the fundamental properties produce, but distinct as regards those fundamental properties themselves. If the properties are causal and if there are two different arrangements of fundamental properties, then there is a difference in their effects, including the way in which they produce the observable phenomena. We may often not be in a position to detect that difference, but it is discernible.

Consider an analogy: whether the world is quantum mechanical or whether it is classical or whether space-time is Galilean (pre-relativistic) or whether it is Lorentzian (special relativity) may be taken to be of little importance when it comes to the description of the macroscopic phenomena that surround us. Nonetheless, there is a slight difference in the description of every macroscopic phenomenon in both these pairs of cases, and that difference can in principle be detected, although our current measuring instruments may often not be in the position to reveal that difference. In other words, we can in principle discover on the basis of the observable phenomena whether we live in a quantum or a classical world and whether we live in a Galilean or a Lorentzian space-time.

Take another example from what is regarded as a common case of multiple realization: genes are functional properties consisting in bringing about certain phenotypic effects via the production of certain proteins, genes are usually realized by certain DNA sequences, and genes of the same type as far as genetics is concerned can be multiply realized by DNA sequences of different molecular types. For instance, there are certain kinds of genes of Escherichia coli, a well-known bacterium in genetic research, coding for the production of proteins that play a predominant role in the flagella, the capsule, or the cell wall. Certain sequences of DNA bring about the proteins in question given normal conditions. These sequences can be of different molecular kinds. Consequently, there are different physical mechanisms to produce the proteins in question. However, these different ways to produce the proteins for which the genes of Escherichia coli code are systematically linked with side effects such as the speed or the accuracy of the protein production (Bulmer 1991). For any such difference in side effects, there is a physical environment possible in which that difference is pertinent to selection and can thus be detected within the scope of classical genetics. 
This line of reasoning applies to any case of multiple realization: whenever there is multiple realization, there are different minimal sufficient physical conditions to bring about the effects that define the multiply realized type. In order to get from one realizer type to another realizer type, one has to replace at least one of the parts that are necessary to bring about the effects in question with a part of another physical type. Against the background of the causal theory of properties, any such replacement implies a systematic difference in the way in which the realizers produce the effects that define the multiply realized type. If there are two or more different ways to bring about these effects, then there is a difference in the production of side effects that are systematically linked with the main effects in question. For any such difference in side effects, there is a physical environment possible in which that difference leads to a detectable difference within the scope of the science of the multiply realized type in question (Esfeld \& Sachse 2007).

Hence, what accounts for quidditism and humility is the categorical character of the fundamental properties, not their supposed intrinsic character. Consequently, an analogous argument applies if one shifts from fundamental, categorical and intrinsic properties to fundamental structures and conceives these structures in a non-modal way, namely as categorical. Again, in that case, the nature of the structures is separated from the causal relations that obtain in a world. Thus, there always is a multiple realizability of the whole domain of causal relations, including the whole domain of observable phenomena, by different types of arrangements of concrete, qualitative fundamental physical structures. In other words, for any possible world $w$ there is another possible world $w^{*}$ such that $w$ and $w^{*}$ are indiscernible as regards the causal relations that obtain in them, but distinct as regards the fundamental structures. We again have to count worlds as different although they are indiscernible, there now being a qualitative difference between fundamental structures that does not make a difference.

By contrast, if the fundamental physical structures are modal structures, being the power to produce certain effects, then, as argued above, any difference in the fundamental structures, accounting for there being two different types of arrangements of fundamental structures in two possible worlds, automatically leads to some difference in the effects that these structures produce and thereby also to some difference in the domain of observable phenomena. That difference can in principle be detected so that there is no principled obstacle from metaphysics to finding out what the fundamental physical structures are.

In a certain sense, the point at issue here is a case of the underdetermination of theoretical entities by observable phenomena. We are confronted with a principled underdetermination if the fundamental properties are categorical (be they intrinsic, be they structures), and that underdetermination disappears in the framework of the causal theory of properties (be the fundamental properties intrinsic, be they structures). If the fundamental properties are categorical and intrinsic, we cannot have any idea of what they are. If the fundamental properties are categorical and structures, one can regard the fundamental physical theories as putting forward conjectures as to what they are by employing mathematical structures. However, we cannot be justified in adopting a realist attitude towards the fundamental structures that any version of the current fundamental physical theories or any successors of them posits, since the whole domain of the observable phenomena of the actual world can be realized by different types of arrangements of fundamental physical structures. Consequently, we can in principle not know what the fundamental physical structures of the actual world 
are. Note the consequence of this argument: the conclusion is not, as in the argument rehearsed in section 2, that our knowledge is limited to causal-cum-structural knowledge and that we cannot know the intrinsic essences underlying the causal-cum-structural relations; instead, the conclusion is that we cannot know the qualitative character of the structures underlying the causal relations including the observable phenomena. That conclusion is incompatible with scientific realism, for it limits our entitlement to claims of knowledge to the domain of the observable phenomena, excluding theoretical entities from what we can know.

That principled underdetermination disappears if one changes the metaphysical framework from the categorical to the causal theory of properties, for differences in the fundamental structures then necessarily lead to causal differences that are in principle detectable. Nonetheless, other, more familiar forms of underdetermination remain untouched by that shift in metaphysics. There always are cases in which a given set of observable phenomena available at a time underdetermines the theoretical entities posited by different theories of the same domain or different interpretations of a given theory. But these are not cases that justify a principled agnosticism as regards the theoretical entities underlying the observed phenomena: the underdetermination at issue in these cases is relative to a given set of observable phenomena at a time, instead of applying to the whole domain of causal relations in a world. For instance, standard quantum mechanics and Bohmian mechanics imply the same observable consequences. However, as soon as one widens the scope to include the observable consequences of quantum field theory (let alone the possible observable consequences of a future theory of quantum gravity), it is unclear whether there can be a Bohmian theory that matches these consequences. The reasoning above is not concerned with such cases of underdetermination, but only with a principled underdetermination arising from the presupposition of the fundamental properties being categorical properties (be they intrinsic, be they structures).

Conceiving the fundamental physical structures in a causal manner has a further advantage: it establishes a clear distinction between mathematical and physical structures. Mathematical structures, whatever they may be, do not cause anything. Real physical structures distinguish themselves from mere mathematical structures in that they are causally efficacious (this point is also stressed by Busch 2003). Hence, if ontic structural realism elaborates on the modal character of the physical structures by conceiving them as causal structures, it avoids the objection of blurring the distinction between the mathematical and the physical at its roots.

In sum, ontic structural realism has been conceived notably by Steven French and James Ladyman as a position that is able to vindicate a certain form of scientific realism with respect to fundamental physics. Against that background, the claim of this paper is that ontic structural realism is suitable as a form of scientific realism only if it commits itself to causal structures, that is to say, only if the essence of the fundamental physical structures is taken to consist in the power to produce certain effects. If, by contrast, the fundamental physical structures are regarded as being categorical, a commitment to scientific realism in the sense of the fundamental physical theories (the current ones, or some future successors of them) being in principle able to reveal the real constitution of the fundamental physical structures is blocked by the fact that the whole domain of causal relations - and consequently the whole domain of observable phenomena - may as well supervene on other fundamental physical structures than those ones admitted by any of the past, current or future physical theories. 
The situation is therefore this one: if one presupposes the metaphysics of categorical properties, science can in principle not give us access to the physical reality underlying the observable phenomena, and quidditism and humility ensue - there are qualitative differences between worlds without those differences being discernible differences. If, by contrast, one abandons that presupposition in favour of the metaphysics of causal properties, no commitment to quidditism or humility follows - any difference between two worlds makes a difference and thus is discernible so that there is no gap between metaphysics and epistemology. Hence, in a nutshell, if ontic structural realism is to be a scientific realism, it has to commit itself to the metaphysics of causal properties in the form of a commitment to the fundamental physical structures being causal structures. (Of course, there are other arguments against scientific realism that are not touched by making the implications of the presupposed metaphysics of properties explicit and that cannot be addressed in this paper; consequently, given that fundamental physics trades in structures, the commitment to causal structures is a necessary but not a sufficient condition for vindicating scientific realism with respect to fundamental physics).

\section{Physics and causal structures}

The considerations in the preceding section applied the debate about categorical vs. causal properties in analytic metaphysics to ontic structural realism, arguing that there is indeed a good reason for ontic structural realism to regard the fundamental physical structures as modal structures in the sense of causal structures. However, does it fit in with physics to conceive the fundamental physical structures - the structures of quantum entanglement, and the spatio-temporal, gravitational structures - in that manner? Ladyman and Ross (2007), for one, have reservations: although they regard the fundamental physical structures as modal structures - without, however, spelling out what exactly their modality is -, they are not inclined to acknowledge causation as a fundamental physical feature. Moreover, starting with Russell (1912), there is a rich tradition in the framework of Humeanism that takes current physics to be incompatible with the causal theory of properties (see notably the papers in Price \& Corry 2007).

Nonetheless, the situation is not as clear as Russell and his contemporary followers suggest. Talk in terms of dispositions is widespread in the interpretation of quantum theory. Entangled states are often conceived as being the disposition to develop into product states, that is, states with classical properties, having definite numerical values that are correlated in a specific way. In that manner, taking quantum states to be dispositions is tied to an interpretation of quantum theory that admits state reductions and thus a transition from quantum properties to really existing classical properties (instead of the world merely appearing to be classical to local observers due to decoherence). The most elaborate physical proposal for such a transition is the one going back to Ghirardi, Rimini and Weber (1986) (GRW) (although there still are a number of physical problems with that proposal that cannot be addressed here, notably the fact that the GRW amendment of the Schrödinger equation to include state reductions does not amount to fully definite numerical values).

The GRW version of quantum theory lends itself to an account in terms of dispositions, conceiving the fundamental physical structures as causal powers: insofar as the structures of quantum entanglement are certain qualitative physical structures, they are the disposition or power - more precisely, the propensity - to produce product states, that is, classical physical 
properties with definite numerical values localized in classical space-time (Dorato 2006, Suárez 2007, 426-433). This disposition is irreducible: it is not grounded on nondispositional, categorical properties. It belongs to the ontological ground floor. It is a real and actual property, not a mere potentiality. It is therefore appropriate to talk in terms of a power for spontaneous localization, this disposition being a causal property that brings about spontaneous localizations. This disposition does not need external manifestation conditions: that is why GRW conceive the disposition in question as one for spontaneous localization. Since entangled states are nonseparable and not localized in space (Howard 1989), it is unproblematic to consider the entangled state as a whole as the disposition or power (propensity) for spontaneous localization.

Conceiving entanglement in that manner has mainly the following four advantages:

(1) It provides for a clear answer to the question what the properties of quantum objects are if there are no properties with definite numerical values, namely dispositions for spontaneous localization in the sense that an entangled state is the disposition for spontaneous localization.

(2) It provides for objective, single case probabilities. It is generally acknowledged that the quantum probabilities cannot be understood in terms of frequencies. Nonetheless, Frigg and Hoefer (2007) prefer a Humean account of the GRW probabilities to an account in terms of propensities. But they do so by relying on the Humean best system virtues of notably simplicity and strength, which, however, are epistemic virtues. By contrast, the account in terms of propensities does not need to have recourse to any epistemic factors. The objective, single case probabilities make true the probabilistic laws in question, which are metaphysically necessary, as mentioned in section 2 above.

(3) It provides for a solution to the measurement problem without invoking observers and without smuggling the notion of measurement into a fundamental physical theory, not being able to clearly define what a measurement is (cf. the case of Schrödinger's cat). There is a power for spontaneous localization in the form of entangled states. The manifestation of this power occurs spontaneously, not requiring interactions and thus being independent of interactions with measurement devices. Measurements are simply one type of interactions among others, which do not call for a special treatment in a fundamental theory of nature.

(4) It accounts for the arrow of time: if there are processes of quantum state reduction, these processes are not time-reversal invariant. Consequently, if the GRW equation or something in its vein is a fundamental physical law, it is a fundamental law that is not time-reversal invariant. The spontaneous localizations that GRW acknowledge can serve as the origin of all time-asymmetric phenomena (Albert 2000, ch. 7). Conceiving the structures of quantum entanglement in terms of dispositions (causal powers) leads to an explanation of this lack of time-reversal invariance: the production of an effect by a cause is the paradigmatic example of an irreversible process. Consequently, if spontaneous localization is the manifestation of a disposition, power or propensity (that is, the power that entangled states are), it is evident why processes of state reduction can in principle not be reversed and why they are the foundation of the arrow of time.

A quantum ontology in terms of dispositions is usually tied to those interpretations of quantum theory that admit state reductions, GRW being the most elaborate of them. If one does not countenance state reductions, one is committed to taking entanglement to be universal and touching all objects including the macroscopic ones and finally the minds of the observers (Albert \& Loewer 1988, Lockwood 1989, ch. 12 and 13). Nevertheless, one has to 
explain the observation of classical properties, including notably the observed measurement outcomes. The most widely accepted solution to that problem consists in saying that entanglement gives rise to a process known as decoherence, and that process accounts for the appearance of a classical physical world to local observers, although entanglement is not reduced. It seems that nothing hinders to conceive the process of decoherence as a causal one such that the structures of entanglement are the power to produce situations (branches of the universe, histories, etc., according to what is one's preferred interpretation within that framework) that appear as classical properties to local observers. Consequently, although a detailed investigation to establish that point still remains to be carried out, as in the interpretations with state reductions, one can take the structures of entanglement to be causal structures, being the power to bring about decoherence and thereby to produce the appearance of a classical world to local observers. If this reasoning proves sound, the view of quantum structures as causal powers turns out to be independent of the stance that one takes on the measurement problem.

Whereas it is hence feasible to spell out the modal character of the quantum structures of entanglement in causal terms, the spatio-temporal relations are widely regarded as the paradigmatic example of non-causal, categorical relations. However, the theory of general relativity teaches us that the metrical field includes the gravitational energy. This theory thereby rules out a dualism of space-time being a passive background arena and matter being inserted into such a space-time. One can regard the metrical field, which includes the gravitational energy, as a physical entity on the same footing as any material entity, and one can take gravitation to be a fundamental physical interaction on a par with the other fundamental physical interactions, such as electromagnetism. On this view, the spatiotemporal, gravitational structures are material structures like the quantum structures of entanglement - although the relations of quantum entanglement and the spatio-temporal, gravitational relations are of course different types of concrete, qualitative physical relations. Against this background, it seems possible to take the spatio-temporal, gravitational relations to be a causal structure like any other material entity: the spatio-temporal, gravitational relations are causal powers (dispositions) that bring about gravitational effects that are in principle observable. Although this interpretation of general relativity still remains to be spelled out in a concrete, detailed manner, it seems thus possible to consider the metrical field as a causal structure (see Bartels 1996, 37-38, and forthcoming as well as Bird forthcoming, sect. 2.3).

In conclusion, let us return to Russell (1912). In his famous attack on the power theory of causation, Russell says: "A volition 'operates' when what it wills takes place; but nothing can operate except a volition. The belief that causes 'operate' results from assimilating them, consciously or unconsciously, to volitions" (Russell 1912,11). Hence, Russell takes the idea of causation as production - and consequently the causal view of properties - to originate in the experience of ourselves as agents in the world, and sees it as an anthropomorphism. The idea of causal properties has indeed its roots in our everyday experience of the world and of ourselves as acting beings. But it is retained in science: the non-physical special sciences such as biology, psychology and the social sciences - trade mainly in functional properties, that is, properties that are defined notably by the effects they bring about or can bring about. In general, we seek for causal explanations in science, and the argument developed in the two preceding sections establishes that if one does not subscribe to a modal conception of the 
properties, including the structures that there are in the world, in the form of the metaphysics of causal properties, including causal structures, one is committed to quidditism and humility, having to accept purely qualitative differences between worlds that are indiscernible. Finally, the brief survey of the current fundamental physical theories in this section shows that it is not only compatible with physics to apply the causal theory of properties to the fundamental physical structures as well, but that there are concrete physical arguments for doing so, notably in the interpretation of quantum theory.

The metaphysics of causal properties holds hence all the way down from common sense including the experience of ourselves as agents in the world via the special sciences to fundamental physics. It therefore provides for a complete and coherent view of the world that reaches from fundamental physics via biology to psychology and to the social sciences. The argument for the metaphysics of causal properties, taking, as physics teaches us, the form of a metaphysics of causal structures, cannot simply be that it is anchored in common sense. The argument is that it leads to a complete and coherent view of the world, including all the domains of empirical science, and avoiding a gap between metaphysics and epistemology by not having to postulate that there is something in the world whose essence is a pure quality that can in principle not be known because it does not make any difference.

\section{Acknowledgements}

I am grateful to two anonymous referees for their comments on the submitted manuscript. For various discussions on the topic of this paper, I would like to thank Andreas Bartels, Dennis Dieks, Mauro Dorato, Steven French, James Ladyman and Holger Lyre as well as my collaborators Vincent Lam, Christian Sachse and Georg Sparber.

\section{References}

ALBERT, D. Z. (2000) Time and chance (Cambridge (Massachusetts), Harvard University Press).

ALBERT, D. Z. \& LOEWER, B. (1988) Interpreting the many worlds interpretation, Synthese, 77, pp. 195-213.

ANJUM, R. L. \& MUMFORD, S. (forthcoming) Dispositional modality, forthcoming in: C. F. GETHMANN

(Ed.) Lebenswelt und Wissenschaft. XXI. Deutscher Kongress für Philosophie, Kolloquien (Hamburg, Meiner).

BARTELS, A. (1996) Modern essentialism and the problem of individuation of spacetime points, Erkenntnis, 45 , pp. $25-43$.

BARTELS, A. (forthcoming) Dispositionen in Raumzeit-Theorien, forthcoming in: C. F. GETHMANN (Ed.) Lebenswelt und Wissenschaft. XXI. Deutscher Kongress für Philosophie, Kolloquien (Hamburg, Meiner).

BEEBEE, H. (2006) Does anything hold the world together?, Synthese, 149, pp. 509-533.

BIRD, A. (2007) Nature's metaphysics. Laws and properties (Oxford, Oxford University Press).

BIRD, A. (forthcoming) Structural properties revisited, forthcoming in: T. HANDFIELD (Ed.) Dispositions and causes (Oxford, Oxford University Press).

BLACKBURN, S. (1990) Filling in space, Analysis, 50, pp. 62-65. Reprinted in S. BLACKBURN (1993) Essays in quasi-realism (Oxford, Oxford University Press), pp. 255-259.

BULMER, M. (1991) The selection-mutation-drift theory of synonymous codon usage, Genetics, 129, pp. 897907.

BUSCH, J. (2003) What structures could not be, International Studies in the Philosophy of Science, 17, pp. 211-223.

CHAKRAVARTTY, A. (2007) A metaphysics for scientific realism: knowing the unobservable (Cambridge, Cambridge University Press). 
DORATO, M. (2006) Properties and dispositions: some metaphysical remarks on quantum ontology, in: A. Bassi, D. Dürr, T. Weber \& N. Zanghi (Eds.) Quantum mechanics: Are there quantum jumps? On the present state of quantum mechanics (American Institute of Physics Conference Proceedings 844) (New York, American Institute of Physics), pp. 139-157.

ELLIS, B. (2001) Scientific essentialism (Cambridge, Cambridge University Press).

ESFELD, M. (2004) Quantum entanglement and a metaphysics of relations, Studies in History and Philosophy of Modern Physics, 35B, pp. 601-617.

ESFELD, M. \& LAM, V. (2008) Moderate structural realism about space-time, Synthese, 160, pp. 27-46.

ESFELD, M. \& SACHSE, C. (2007) Theory reduction by means of functional sub-types, International Studies in the Philosophy of Science, 21, pp. 1-17.

FLORIDI, L. (2008) A defence of informational structural realism, Synthese, 161, pp. 219-253.

FRENCH, S. (2006) Structure as a weapon of the realist, Proceedings of the Aristotelian Society, 106, pp. $167-$ 185.

FRENCH, S. \& LADYMAN, J. (2003) Remodelling structural realism: quantum physics and the metaphysics of structure, Synthese, 136, pp. 31-56.

FRIGG, R. \& HOEFER, C. (2007) Probability in GRW theory, Studies in History and Philosophy of Modern Physics, 38B, pp. 371-389.

GHIRARDI, G. C., RIMINI, A. \& WEBER, T. (1986) Unified dynamics for microscopic and macroscopic systems, Physical Review, D34, pp. 470-491.

HANDFIELD, T. (2008) Humean dispositionalism, Australasian Journal of Philosophy, 86, pp. 113-126.

HAWTHORnE, J. (2001) Causal structuralism, Philosophical Perspectives, 15, pp. 361-378.

HEIL, J. (2003) From an ontological point of view (Oxford, Oxford University Press).

HEIL, J. (2009) Obituary: C. B. Martin, Australasian Journal of Philosophy, 87, pp. 177-179.

HOFFMANN, V. (2008) The metaphysics of extrinsic properties. An investigation of the intrinsic / extrinsic distinction and the role of extrinsic properties in the framework of physicalism (University of Bonn, PhD Thesis).

HOWARD, D. (1989) Holism, separability, and the metaphysical implications of the Bell experiments, in: J. T. CUSHING \& E. McMULLIN (Eds.) Philosophical consequences of quantum theory. Reflections on Bell's theorem (Notre Dame, University of Notre Dame Press), pp. 224-253.

JACKSON, F. (1998) From metaphysics to ethics. A defence of conceptual analysis (Oxford, Oxford University Press).

LADYMAN, J. (1998) What is structural realism?, Studies in History and Philosophy of Modern Science, 29, pp. $409-424$.

LADYMAN, J. \& ROSS, D. with SPURRETT, D. \& COLLIER, J. (2007) Every thing must go. Metaphysics naturalised (Oxford, Oxford University Press).

LANGTON, R. \& LEWIS, D. (1998) Defining 'intrinsic', Philosophy and Phenomenological Research, 58, pp. 333-345. Reprinted in D. LEWIS (1999), Papers in metaphysics and epistemology (Cambridge: Cambridge University Press), pp. 116-132.

LEWIS, D. (1986) Philosophical papers. Volume 2 (Oxford, Oxford University Press).

LEWIS, D. (2009) Ramseyan humility, in: D. BRADDON-MITCHELL \& R. NOLA (Eds.) Naturalism and analysis (Cambridge (Massachusetts), MIT Press), pp. 203-222.

LOCKE, D. (2009) A partial defense of Ramseyan humility, in: D. BRADDON-MITCHELL \& R. NOLA (Eds.) Naturalism and analysis (Cambridge (Massachusetts), MIT Press), pp. 223-241.

LOCKWOOD, M. (1989) Mind, brain and the quantum. The compound ' $I$ ' (Oxford, Blackwell).

MARTIN, C. B. (1997) On the need for properties: the road to Pythagoreanism and back, Synthese, 112, pp. 193-231.

MUMFORD, S. (2006) The ungrounded argument, Synthese, 149, pp. 471-489.

NEY, A. (2007) Physicalism and our knowledge of intrinsic properties, Australasian Journal of Philosophy, 85 , pp. 41-60. 
PRICE, H. \& CORRY, R. (Eds.) (2007) Causation, physics, and the constitution of reality. Russell's republic revisited (Oxford, Oxford University Press).

PSILLOS, S. (2006) The structure, the whole structure and nothing but the structure, Philosophy of Science, 73 , pp. $560-570$.

RUSSELL, B. (1912) On the notion of cause, Proceedings of the Aristotelian Society, 13, pp. 1-26.

SHOEMAKER, S. (1980) Causality and properties, in: P. VAN INWAGEN (Ed.) Time and cause (Dordrecht, Reidel), pp. 109-135. Reprinted in S. SHOEMAKER (1984), Identity, cause, and mind. Philosophical essays (Cambridge, Cambridge University Press), pp. 206-233.

SLOWIK, E. (2005) Spacetime, ontology, and structural realism, International Studies in the Philosophy of Science, 19, pp. 147-166.

SPARBER, G. (2008) Unorthodox Humeanism (University of Lausanne, PhD Thesis; forthcoming Frankfurt (Main), Ontos Verlag).

SUAREZ, M. (2007) Quantum propensities, Studies in History and Philosophy of Modern Physics, 38B, pp. 418-438.

WHITTLE, A. (2006) On an argument for humility, Philosophical Studies, 130, pp. 461-497. 